\title{
VEHICLE TRACKING USING KALMAN FILTER AND FEATURES
}

\author{
Amir Salarpour ${ }^{1}$ and Arezoo Salarpour ${ }^{2}$ and Mahmoud Fathi ${ }^{2}$ and MirHossein \\ Dezfoulian $^{1}$ \\ ${ }^{1}$ Department of Computer Engineering, BuAliSina University, Hamedan, Iran \\ \{a.salarpour, dezfoulian\} @basu.ac.ir \\ ${ }^{2}$ Department of Computer Engineering, Iran University of Science and \\ Technology, Tehran, Iran \\ arezoo.salarpour@gmail.com, mahfathi@iust.ac.ir
}

\begin{abstract}
Vehicle tracking has a wide variety of applications. The image resolution of the video available from most traffic camera system is low. In many cases for tracking multi object, distinguishing them from another isn't easy because of their similarity. In this paper we describe a method, for tracking multiple objects, where the objects are vehicles. The number of vehicles is unknown and varies. We detect all moving objects, and for tracking of vehicle we use the kalman filter and color feature and distance of it from one frame to the next. So the method can distinguish and tracking all vehicles individually. The proposed algorithm can be applied to multiple moving objects.
\end{abstract}

\section{KEYWORDS}

Kalman filter, occlusion, active contour

\section{INTRODUCTION}

There are a number of traffic monitoring technologies being used. Traffic cameras provide a more flexible way of monitoring traffic. These cameras not only can be used in simple tasks like counting cars, they also have the potential to be used in more complex applications like tracking.

Multiple object tracking is an important research topic in computer vision. It has the ability of deal with the single object difficulties such as occlusion with background changing appearance, illumination, non rigid motion and the multi object difficulties such as occlusion between objects and object confusion. In [1] tracking fix number of objects. In [2] an efficient algorithm to track multiple people is presented. [3] proposed a bayesian tracker for tracking multiple blob. Various tracking algorithms have been proposed in the literature, including approaches templates and local features [4] Kalman filters [13][14] and contours [11][12]. The mean-shift algorithm was first adopted as an efficient tracking technique in [15].

In tracking systems two problems must be considered: prediction and correction.

Predict problem: predict the location of an object being tracked in the next frame, that is identify a region in which the probability of finding object is high.

Correction problem: identify the object in the next frame within designated region.

A well-known solution for prediction is Kalman filter, a recursive estimator of state of a dynamic system. Kalman filter have been adopted in video tracking [5]. To predict the search region more effectively, mean-shift was combined with Kalman filter in [16]. To improve the ability of mean-

DOI : 10.5121/sipij.2011.2201 
shift tracking with respect to scale changes, a scale selection mechanism was introduced in [17] based on Lindeberg's theory. Mean-shift has been used in the past to track moving targets in sequences of FLIR imagery [18] and human bodies (i.e., non-rigid objects) in [19].

The correction problem requires a similarity metric to compare candidate pairs of object in previous and current frame. This is the correspondence of object in two frames. Matching metrics in correction problem is important. A tracking problem is data association, finding the true position of moving target, when there are more than one valid candidate. This occurs in clutter scenes.

The success or failure of any tracking algorithm depends a lot on the degree that the tracked object can be distinguished from its surroundings [10]. In particular, the set of features used by the tracking algorithm to represent the object(s) being tracked plays a major role in tracking performance.

Using color information alone is not sufficient for on-road vehicle tracking. Competing clutters having similar colors as the target may appear in the background quite often. Moreover, the color of the target being tracked varies due to light changes and road conditions.

Consequently, robust tracking performance can be gained by switching to shape information when color information becomes less reliable and the opposite.

The reminder of this paper is as follows: section 2 is a concise review of video tracking algorithms in computer vision. In section 3 we describe our approach. And in section 4 we present the results of multiple vehicle tracking. And the section 5 devoted to the conclusion.

\section{TRACKING METHODS}

Tracking methods can be divided into four major categories: region based, model based, active contour based and feature based.

Region based algorithms track objects according to variations of the regions corresponding to the moving objects [6,7]. These algorithms usually detect motion region by subtracting the background from the current image. These algorithms cannot work well when there are multiple moving objects. The idea in region- or blob-based tracking is to identify connected regions of the image, blobs, associated with each vehicle. Regions are often obtained

through background subtraction, for which many methods exist, and then tracked over time using information provided by the entire region [20][21].

Model based algorithms track objects by matching object model, which produced later [8]. There are one or several models of vehicles. They are most robust than the feature based algorithms but slower.

Active contour tracking algorithms track objects by their contours. They update these contours in consecutive frames [9]. These algorithms provide more efficient description of objects than do region-based algorithms, and the computational complexity is reduced. However, the inability to segment vehicles that are partially occluded remains. If a separate contour could be initialized for each vehicle, the tracking could be done even in the presence of partial occlusion. For all methods, initialization is one of the major problems.

Feature based algorithms track objects by extracting their features and matching these features between frames [8][22][23].

There are some features that define a vehicle (symmetry, edges, shadow, color, size), and they are looked for sequentially in the image.

We use both region based and feature based tracking in this work. 


\section{OURMETHOD OBJECTDETECTION}

\subsection{Background Estimation}

The proposed method uses both region based and feature based tracking algorithms for tracking cars. For prediction step we use region based and predict the position of object in frame at time $\mathrm{t}+1$.

We consider the center of the object as a point. Then we use Kalman filter in frame at time $t$ for predict the position of this point in frame at time $t+1$. then we use the distance and color feature for correction in frame $t+1$, we match the color of vehicle and check its distance. And we find if it predicts correctly or not. If it has errors, this step corrects the errors.

First we should detect vehicles, we use an adaptive background model.

In this section first we describe an adaptive background generation and moving objects detection. Then the tracking algorithm is described.

Notice that in this article camera position assumed stationary. At first, background frame B0(x, y) is initialized by the first frame of image sequence.

$$
B_{0}(x, y)=I_{0}(x, y)
$$

For determined changes in frame mask $M_{n}(x, y)$ is defined by thresholding the difference between three consecutive frames.

$$
M_{n+1}(x, y)=\left\{\begin{array}{cc}
0 & f\left|\mathrm{I}_{\mathrm{n}+1}(\mathrm{x}, \mathrm{y})-\mathrm{I}_{\mathrm{n}}(\mathrm{x}, \mathrm{y})\right|<1 \text { or } \\
& \left|\mathrm{I}_{\mathrm{n}+1}(\mathrm{x}, \mathrm{y})-\mathrm{I}_{\mathrm{n}-1}(\mathrm{x}, \mathrm{y})\right|<1, \\
1 & \text { otherwise }
\end{array}\right.
$$

Now adaptive background will created by $M_{n}(x, y)$. The background update model for $(\mathrm{n}+1)$ frames is defined as follow:

$$
\mathrm{B}_{\mathrm{n}+1}(\mathrm{x}, \mathrm{y})=\left\{\begin{array}{cl}
\alpha \mathrm{B}_{\mathrm{n}}(\mathrm{x}, \mathrm{y})+(1-\alpha) \mathrm{I}_{\mathrm{n}}(\mathrm{x}, \mathrm{y}) & \text { if } \mathrm{M}_{\mathrm{n}}(\mathrm{x}, \mathrm{y})=0 \\
\mathrm{~B}_{\mathrm{n}}(\mathrm{x}, \mathrm{y}) & \text { otherwise }
\end{array}\right.
$$

Where $\alpha \in 0,1$ is a time constant that controls the rate of the adaptation of the background. In the other word percentage of changed pixels in the current frame is defined whit respect to the previous two frames. To define the best value for $\alpha$ we suggest following equation:

$$
\alpha=\frac{\sum M_{n}(x, y)}{\text { areaof } I_{n}}
$$




\subsection{Object Detection}

The most used approach to detect objects in a video sequence is background subtraction. There are situations for which a poor implementation of the scheme causes an erroneous/coarse segmentation, so that consequent processes of tracking and recognition often fail. An automatic approach preserves from such inconvenience. We assume $B(x, y), I(x, y)$ the gray-valued frame and the corresponding adaptive background model. A binary mask $D_{n}(x, y)$ is performed to segment out objects of interest (foreground detection)

$$
D_{n}(x, y)=\left\{\begin{array}{lc}
0 & \text { if } S_{n}(x, y) \leq T h \\
1 & \text { otherwise }
\end{array}\right.
$$

Where $S_{n}(x, y)$ and then compute whit following relations:

$$
\begin{gathered}
S_{\mathrm{n}}(\mathrm{x}, \mathrm{y})=\left|\mathrm{I}_{\mathrm{n}}(\mathrm{x}, \mathrm{y})-\mathrm{B}_{\mathrm{n}}(\mathrm{x}, \mathrm{y})\right| \\
\mathrm{MED}=\operatorname{median}\left[\mathrm{S}_{\mathrm{n}}(\mathrm{x}, \mathrm{y})\right] \quad \forall(\mathrm{x}, \mathrm{y}) \in \mathrm{I}_{\mathrm{n}}, \\
\mathrm{MAD}=\operatorname{median}\left[\mathrm{S}_{\mathrm{n}}(\mathrm{x}, \mathrm{y})-\mathrm{MED}\right] \quad \forall(\mathrm{x}, \mathrm{y}) \in \mathrm{I}_{\mathrm{n}}
\end{gathered}
$$

We will have an adaptive method according the above relations defined.

\section{OBJECT TRACKING}

We use both region based and feature based algorithms for tracking. First for region based we use the Kalman filter for find the region of vehicle in the next frame. We find the center of object, then use Kalman filter for predict the position of it in the next frame.

\subsection{Kalman Filter}

A Kalman filter is used to estimate the state of a linear system where the state is assumed to be distributed by a Gaussian. Kalman filtering is composed of two steps, prediction and correction. The prediction step uses the state model to predict the new state of the variables:

$$
\begin{gathered}
\bar{X}^{t}=D X^{t-1}+W \\
\bar{\Sigma}^{t}=D \sum^{t-1} D^{T}+Q^{T}
\end{gathered}
$$

Where and are the state and the covariance predictions at time $t$. $\mathbf{D}$ is the state transition matrix which defines the relation between the state variables at time $t$ and $t-1 . Q$ is the covariance of the noise $W$. Similarly, the correction step uses the current observations to update the object's state:

$$
X^{t}=\bar{X}^{t}+K^{t}\left[Z^{t}-M \bar{X}^{t}\right]
$$


Signal \& Image Processing : An International Journal (SIPIJ) Vol.2, No.2, June 2011

$\mathbf{M}$ is the measurement matrix, $K$ is the Kalman gain. Note that the updated state, is still distributed by a Gaussian. Kalman filter, the extended Kalman filter assumes that the state is distributed by a Gaussian.

\subsection{Correction: Color and size features}

After using the displacement of vehicle, and predicting the position of vehicle in the next frame, the next step is to correct the results of prediction step. For the correction step we use the feature based method.

Now we insert two conditions on color and size features for corresponding point.

The first condition is the comparison of blobs color in two consecutive frames. The color of blob in frame $\mathrm{n}$ compared with the color of blobs in $\mathrm{n}+1$ frame, and it must be the same.

The second condition is on the size of blobs in two frames.

Since that the distance between vehicle and camera is varying, the size of vehicle is varying too. But from one frame to the next the variation ratio in size is low, and the difference between sizes of two blobs in consecutive frame must be low.

So if the colors of two blobs be matched and the size difference between them be lower than a threshold, and the predicted choice satisfies these two conditions, we consider that the prediction is true and set the predicted position to center of blob in new frame.

In this condition if two vehicles are close to each other, with deal with these conditions the correct choice is selected for each vehicle. Also if the occlusion is occurred, after that with match the condition each vehicle can be tracked correctly.

\section{RESULTS}

The proposed tracker has been tested under some tracking videos. Two evaluate the performance of our method we use the accuracy measure. The average accuracy of proposed method is $96 \%$.

\subsection{Tracking Performance Evaluation}

Point tracking methods can be evaluated on the basis of whether they generate correct point. Given a ground truth, the performance can be evaluated by computing accuracy measure. In the context of point tracking, the accuracy can be defined as:

$$
\text { Accuracy }=\frac{\text { number of correct correspondence }}{\text { number of correspondence }}
$$

correct correspondence is the number of points which are detected and tracked correctly by the proposed method. And the correspondence term is the total number of correspondence points.

\subsection{Experimental Results}

In this section we present our method on real image sequence. The algorithm acts correctly in clutter scene that is presented in figures. Multiple vehicles can be tracked simultaneously.It can control some problem of multi object tracking, such as appearance and disappearance of objects, and missing of a vehicle. 
Signal \& Image Processing : An International Journal (SIPIJ) Vol.2, No.2, June 2011
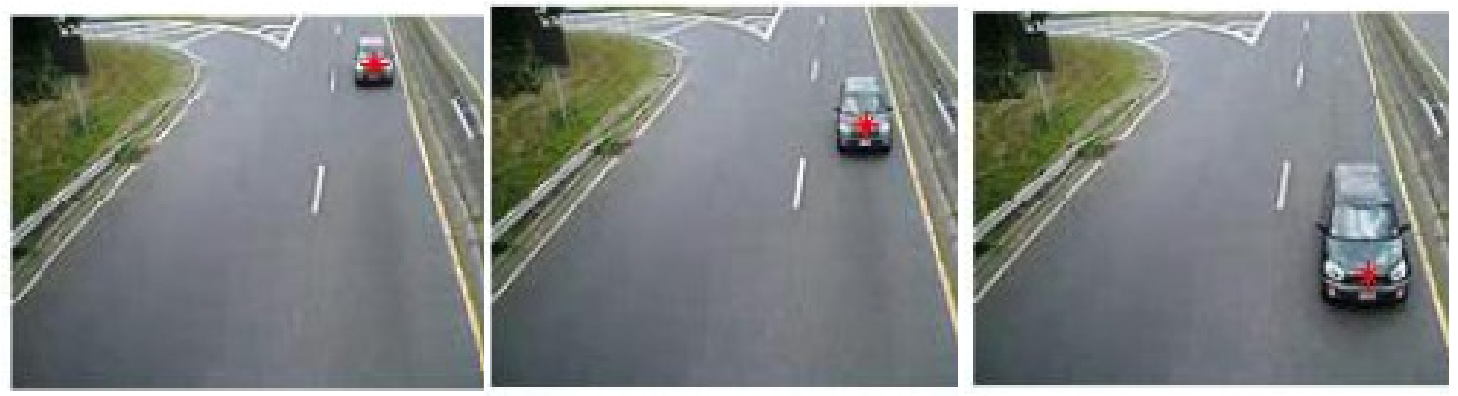

Figure 1. Multi car tracking

\section{CONCLUSIONS}

In this paper we presented a tracking method for processingvideo data in order to perform tracking by a machine vision system. The objective of the presented approach is to minimize the computation time, and use the feature of vehicles for the best tracking.

The outcome of our approach is the acquisition of route related data such as travel times and tracing of traffic streams, which can highly increase the efficiency of traffic control systems.
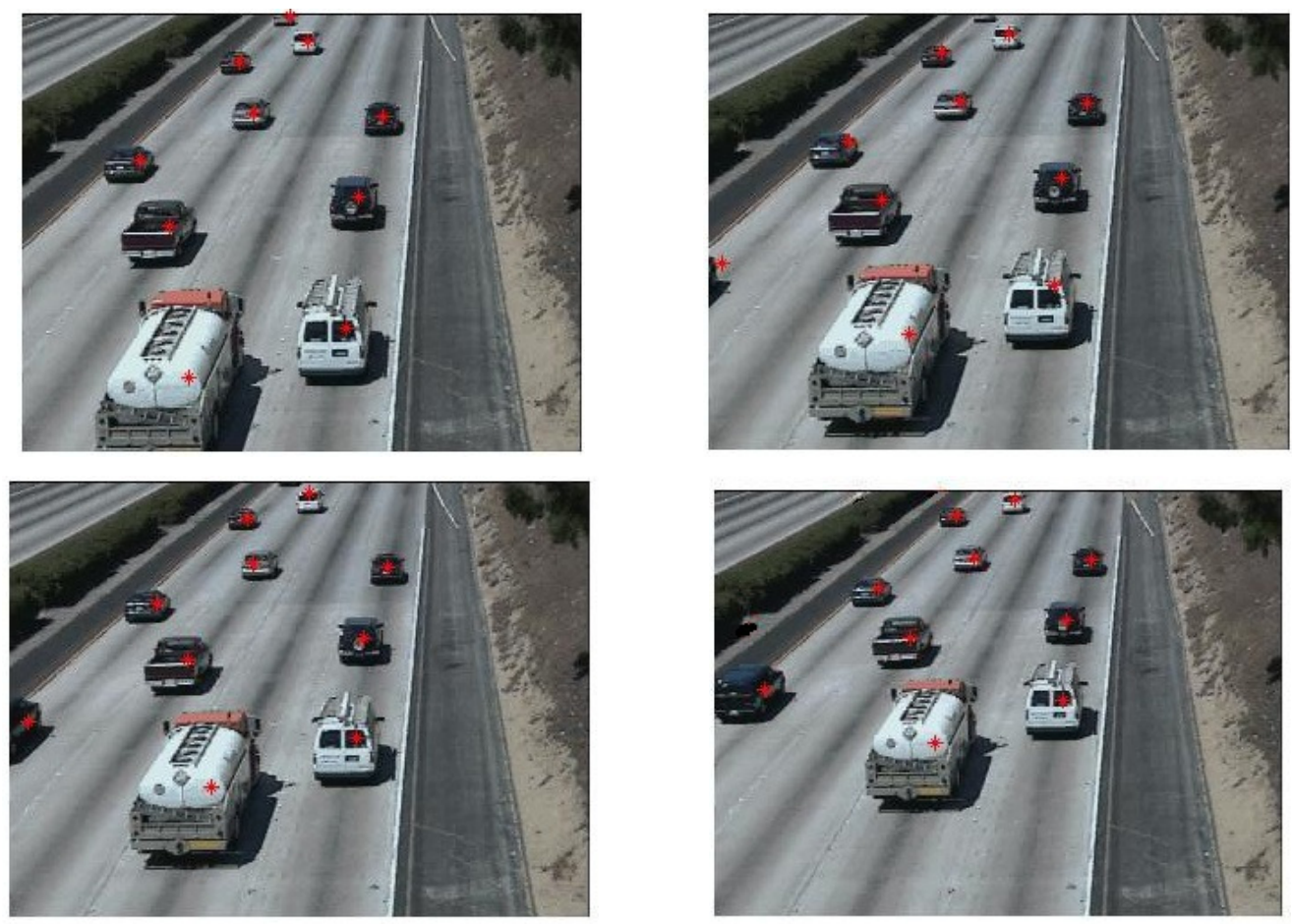

Figure 2. Multi car tracking in clutter scene 
Signal \& Image Processing : An International Journal (SIPIJ) Vol.2, No.2, June 2011

In this article we presented a combinational method for tracking multiple vehicles. This method is combined of two categories of algorithms. Region based and feature based. First we use displacement of vehicle for predict the position of it in the next frame, then we use two features of vehicle,color and size to make correspondence.

The proposed algorithm can deal with the tracking problem such as appearance, disappearance and occlusion. It can work in clutter scene and the results are satisfactory.

\section{REFERENCES}

[1] J.P. MacCormick and A. Blake, (1999) "A probabilistic exclusion principle for tracking multiple objects", in ICCV99, 572-578.

[2] H. Tao, H.S. Sawhney and R. Kumar, (1999) "A sampling algorithm for tracking multiple objects", in Vision Algorithms.

[3] C. Hue, J.P. Le Cadre and P.Perez, (2002) "Tracking multiple objects with particle filtering", IEEE Transactions on Aerospace and Electronic Systems, 791-812.

[4] Y. Raja, S. J. McKenna and S. Gong, (1998) "Segmentation and tracking using color mixture model", Asia Conference on Computer Vision.

[5] G. S. Manku, P. Jain, A. Aggarwal and L. Kumar, (1997) "Object tracking using affine structure for point correspondence", IEEE conference of Computer Vision and Pattern Recognition, 704709.

[6] P. Kumar, H. Weimin, I. U. Gu and Q. Tian, (2004) "Statistical modeling of complex backgrounds for foregroundobject detection", IEEE Transaction on Image Processing, 43-53.

[7] Boyoon Jung and Gaurav S. Sukhatme, (2004) "A Generalized Region-based Approach for Multitarget Tracking in Outdoor Environments", IEEE International Conference on Robotics and Automation, 2189-2195.

[8] D. Serby, E. K. Meier and L. V. Gool, (2004) "Probabilistic Object Tracking Using Multiple Features", IEEEPattern Recognition Intelligent Transportation Systems, 43-53.

[9] Zhimin Fan, Jie Zhou, Dashan Gao and Zhiheng Li, (2002) "Contour Extraction And Tracking Of Moving Vehicles For Traffic Monitoring”, IEEE International Conference on Intelligent Transportation Systems, 84-87.

[10] Robert T. Collins and Yanxi Liu, (2003) "On-line selection of discriminative tracking features," IEEE International Conference on Computer Vision, vol. Nice, France, pp. 346-352.

[11] Esther B. Meier and Frank Ade, (1999) "Using the condensation algorithm to implement tracking for mobile robots," Third European Workshop on Advanced Mobile Robots, pp. 73-80, Zurich, Awitzerland.

[12] Andrea Giachetti, “Applications of contour tracking techniques," citeseer.ist.psu.edu/7949.html.

[13] J Lou, H Yang,Wei Ming Hu, Tieniu Tan, (2002) "Visual vehicle tracking using an improved ekf," Asian Conference on Computer Vision.

[14] D. Koller, J. Weber, and J. Malik, (1994) "Robust Multiple Car Tracking with Occlusion Reasoning”.Third European Conference on Computer Vision, pp. 186-196, Springer-Verlag. 
Signal \& Image Processing : An International Journal (SIPIJ) Vol.2, No.2, June 2011

[15] D. Comaniciu, V. Ramesh and P. Meer, (2002) "Real-time tracking of non-rigid objects using mean shift," IEEE Conf. on Computer Vision and Pattern Recognition, vol. 2, pp. 142-149, Hilton Head Island, South Carolina.

[16] D. Comaniciu and V. Ramesh, (2000) "Mean shift and optimal predication for efficient object tracking”, IEEE Int. Conf. Image Processing, vol. 3, pp. 70-73, Vancouver,Canada.

[17] Robert T. Collins, (2003) "Mean-shift blob tracking through scale space," IEEE Computer Vision and Pattern Recognition, vol. Madison, WI, pp. 234-240.

[18] AlperYilmaz, KhurramShafique, NielsLovo, Xin Li, Teresa Olson, Mubarak A. Shah, (2001) "Target tracking in flir imagery using mean-shift and global motion compensation," proceedings of IEEE Workship on Computer Vision Beyond Visible Spectrum, Hawaii.

[19] FatihPorikli and OncelTuzel, (2003) "Human body tracking by adaptive background models and mean-shift analysis," IEEE International Workshop on Performance Evaluation of Tracking and Surveillance.

[20] D. Magee, (2004) "Tracking multiple vehicles using foreground, background and motion models". Image and Vision Computing, 22:143-155.

[21] B. Maurin, O. Masoud, and N. P. Papanikolopoulos, (2005)“Tracking all traffic: computer vision algorithms for monitoring vehicles, individuals, and crowds”, Robotics \& Automation Magazine, IEEE, 12(1):29-36.

[22] S. T. Birchfield, (1999) "Depth and Motion Discontinuities",PhD thesis, Stanford University.

[23] N. K. Kanhere, S. J. Pundlik, and S. T. Birchfield, (2005) "Vehicle segmentation and tracking from a low-angle off-axis camera", In IEEE Conference on Computer Vision and Pattern Recognition, San Diego, California. 